\title{
Exisfence And Uniqueness Of Solufion For Fractional Integro Differential Equation With Boandary Conditions
}

\author{
N. A. Abdul - Kader \\ N. L. Housen \\ N. A. Abdul-Razaq \\ Department of Mathematics / College of Education for pure Science \\ University of Mosul / Mosul - Iraq. \\ mahmod.amir74@gmail.com Intropy77@yahoo.com adnan.razzaq@gmail.com
}

DOI: $\underline{10.33899 / \text { edusj.2019.162968 }}$

Received

03 / 01 / 2019
Accepted

27 / 01 / 2019

\begin{abstract}
Our work investigates the Existence and Uniqueness of the solution of nonlinear fractional integro-differential equation with boundary Conditions by applying fixed point theorems.
\end{abstract}

Keywords: Fractional integro-differential equation, Existence and Uniqueness solutions, Baundary conditions. 


\section{وجود ووحدانية الحل لمعادلة تكاملية- تفاضلية من الرتبة الكسرية ذات شروط حدودية}

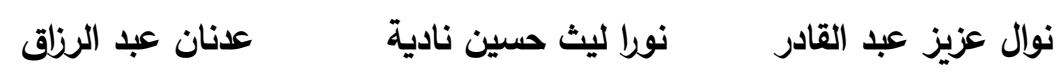

$$
\begin{aligned}
& \text { قسم الرياضيات / كلية التربية للعلوم الصرفة }
\end{aligned}
$$

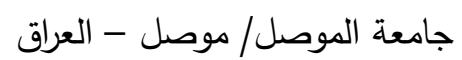

adnan.razzaq@gmail.com Intropy77@yahoo.com mahmod.amir74@gmail.com

DOI: $\underline{10.33899 / \text { edusj.2019.162968 }}$

القبول

2019 / 01 / 27
الاستلام

2019 / 01 / 03

الملخص

خلال هذا البحث قدمنا بعض المبرهنات المتعلقة بوجود ووحدانية الحل لمعادلة تكاملية تفاضلية من

الرتبة الكسرية ذات شروط حدودية بالاعتماد على مبرهنات النقطة الثابتة.

الكلمات المفتاحية: معادلة تكاملية-تفاضلية كسرية، وجود ووحدانية الحل، شروط حدودية.

\section{1- المقدمة}

في الآونة الاخيرة، تعد المعادلات التفاضلية الكسرية ذات أهمية كبيرة لعلماء الرياضيات، اذ كانت المفية السبب في تطوير نظرية حسبان التفاضل والتكامل الكسري، فضلاً عن تطبيقات الانكماش في مختلف العلوم

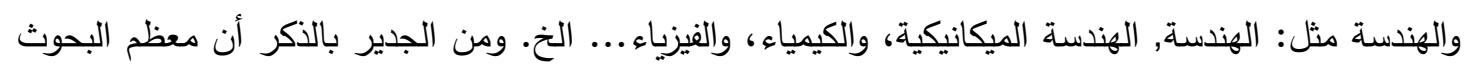

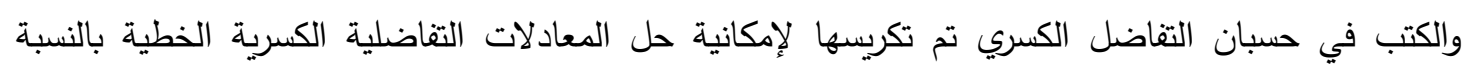
للدوال الخاصة.

نُشر مؤخراً بعض الابحاث التي تتتاول وجود وتعدد الحلول (أو الحل الموجب) للمعادلة التفاضلية

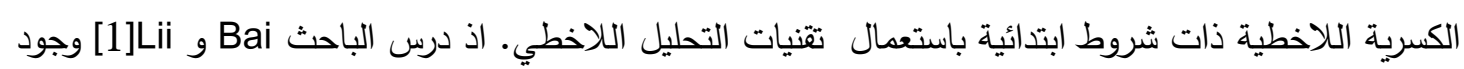
وتعدد الحلول الموجبة لمسائل القيمة الحدودية للمعادلة التفاضلية الكسرية اللاخطية, وفي السنوات الاخيرة

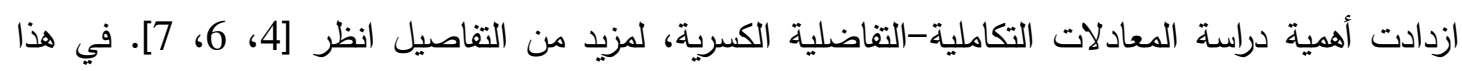
البحث تم اعتماد المعادلة التكاملية-التفاضلية التالية:

$\left.{ }^{c} D^{q} \phi(t)=\psi\left(t, \phi(t), \int_{0}^{t} k(t, y, \phi(y)) d y, \int_{0}^{T} g(t, y, \phi(y)) d y\right)\right)$

ذات الشروط الحدودية

$\phi(0)=\delta \neq 0, \quad \phi^{\prime}(T)=\gamma \neq 0$ 


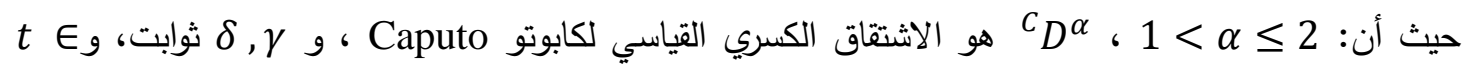

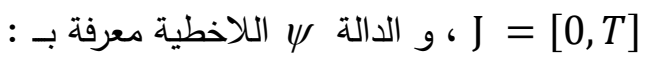

$\psi:[0, T] \times \bar{X} \times \bar{X} \times \bar{X} \rightarrow \bar{X}$

$k, g: J \times J \times \bar{X} \rightarrow \bar{X}$

حيث أن X Xو فضاء باناخ Banach مزود بمعيار || .|| .

$C=C([0, T], \bar{X})$

لتكن

$P:[0, T] \rightarrow \bar{X}$

فضاء باناخ لكل الدوال المستمرة

-

يهدف هذا البحث الى دراسة وجود الحل لمسألة القيمة الحدودية (1.2)-(1.1) لمعادلة تكاملية-

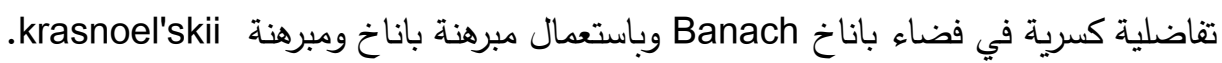

2- تعريفات

في هذا البند سنقدم بعضا من التعريفات والمأخوذات والمبرهنات التي نحتاجها في عملنا هذا.

[2، [2.1) تعريف [-

اذا كانت

${ }^{b} \mathrm{I}_{a}^{\alpha} f=\frac{1}{\Gamma(\alpha)} \int_{a}^{b}(b-s)^{\alpha-1} f(s) d s$.

وبشرط وجود التكامل اعلاه.

[2 [2.2) تعريف -

لتكن f دالة معرفة على الفترة المغلقة [a , b]، فأن الاشتقاق الكسري لكابوتو Caputo يعرف بالصيغة

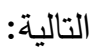

$\left({ }^{c} D_{0}^{\alpha} f\right)(t)=\frac{1}{\Gamma(n-\alpha)} \int_{0}^{t}(t-s)^{n-\alpha-1} f^{(n)}(s) d s$.

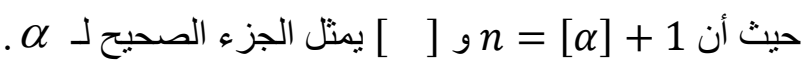

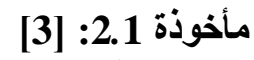

${ }^{t} D_{a}^{-\alpha}{ }^{t} D_{a}^{\alpha} x(t)=x(t)+c_{0}+c_{1} t+c_{2} t^{2}+\cdots c_{n-1} t^{n-1}$.

لتكن 0 ف

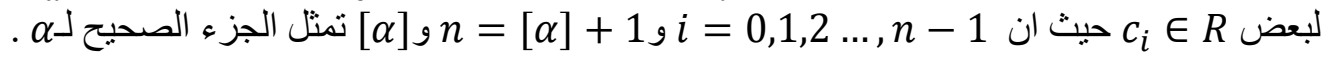

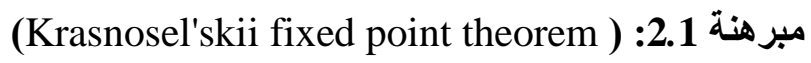

لتكن M مجموعة جزئية غير خالية من فضاء باناخ

وليكن R و Sؤثرين بحيث أن: 


$$
\begin{aligned}
& \text {.x, } y \in M \text { S } R \text { ، لكل } \\
& \text { R-2 } \\
& \text { · } 3-3
\end{aligned}
$$

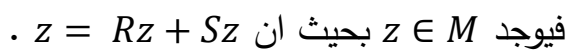

ليكن

$$
\text { والدالة }
$$

افرض المعادلة التكاملية-التفاضلية الكسرية ذات الشروط الحدودية التي لها الصيغة التالية: $\left.{ }^{c} D^{q} \phi(t)=\psi\left(t, \phi(t), \int_{0}^{t} k(t, y, \phi(y)) d y, \int_{0}^{T} g(t, y, \phi(y)) d y\right)\right)$

$\phi(0)=\delta \neq 0 \quad, \phi^{\prime}(T)=\gamma \neq 0$.

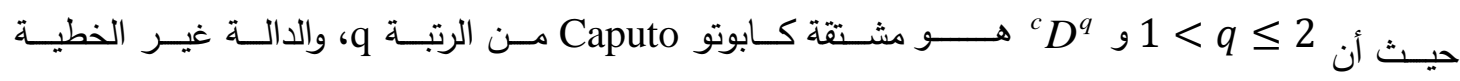
: $k$ : $20, T] \times[0, T] \times \bar{X} \rightarrow \bar{X}, \psi:[0, T] \times \bar{X} \times \bar{X} \times \bar{X} \rightarrow \bar{X}$ أ- يوجد دوال مستمرة

$\left\|\int_{0}^{t}(k(t, r, x)-k(t, r, y)) d r\right\| \leq h(t)\|x-y\|$

$\left\|\int_{0}^{t} k(t, r, y) d r\right\| \leq h_{1}(t)\|y\|$

لكل

ب- وجود دوال مستمرة

$\left\|\int_{0}^{T}(g(t, r, x)-g(t, r, y)) d r\right\| \leq u(t)\|x-y\|$

9

$\left\|\int_{0}^{T} g(t, r, y) d r\right\| \leq u_{1}(t)\|y\|$

$. x, y \in \bar{X} \quad, \quad t, r \in[0, T]$ لكل

ج- يوجد دوال مستمرة $\rightarrow R^{+}$ $\left\|\psi\left(t, x_{1}, y_{1}, z_{1}\right)-\psi\left(t, x_{2}, y_{2}, z_{2}\right)\right\| \leq m(t) Q\left(\left\|x_{1}-x_{2}\right\|+\left\|y_{1}-y_{2}\right\|+\left\|z_{1}-z_{2}\right\|\right)$.

$\mathrm{N}^{*}=\sup _{\mathrm{t} \in[0, \mathrm{~T}]}\|\psi(t, 0,0,0)\|$

لكل [0, . $\mu:[0, T] \rightarrow R^{+}=[0, \infty)$. 


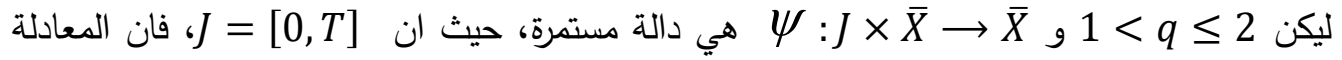
التفاضلية الكسرية (1.1) ذات الشروط الحدودية (1.2) تكافئ المعادلة التكاملية $\phi(t)=\delta+\gamma t+\frac{1}{\Gamma(q)} \int_{0}^{t}(t-y)^{q-1} \psi\left(y, \phi(y), \int_{0}^{y} k(y, \sigma, \phi(\sigma)) d \sigma, \int_{0}^{T} g(y, \sigma, \phi(\sigma)) d \sigma\right) d y$ $-\frac{t}{\Gamma(q-1)} \int_{0}^{T}(T-y)^{q-2} \psi\left(y, \phi(y), \int_{0}^{y} k(y, \sigma, \phi(\sigma)) d \sigma, \int_{0}^{T} g(y, \sigma, \phi(\sigma)) d \sigma\right) d y$

البرهان: من المأخوذة (2.1)، بالإمكان تحويل المسألة (1.2)-(1.1) لتكافئ المعادلة التكاملية التالية: $\phi(t)={ }_{0}^{t}{ }_{0}^{q} \psi+c_{1}+c_{2} t$.

$\phi(t)=\frac{1}{\Gamma(q)} \int_{0}^{t}(t-y)^{q-1} \psi\left(y, \phi(y), \int_{0}^{y} k(y, \sigma, \phi(\sigma)) d \sigma, \int_{0}^{T} g(y, \sigma, \phi(\sigma)) d \sigma\right) d y+c_{1}+c_{2} t$.

وفي ضوء العلاقة

${ }^{t} D^{q} I^{q} \phi(y)=\phi(y), I^{q} I^{\beta} \phi(t)=I^{q+\beta} \phi(t)$.

لكل 0 >

$\phi^{\prime}(t)=\frac{1}{\Gamma(q-1)} \int_{0}^{t}(t-y)^{q-2} \psi\left(y, \phi(y), \int_{0}^{y} k(y, \sigma, \phi(\sigma)) d \sigma, \int_{0}^{T} g(y, \sigma, \phi(\sigma)) d \sigma\right) d y+c_{2} t$

$\phi(0)=c_{1}=\delta$

$\phi^{\prime}(T)=\mathrm{I}^{q-1} \psi+c_{2}=\gamma$

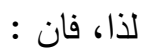

$c_{2}=\gamma-\frac{1}{\Gamma(q-1)} \int_{0}^{T}(T-y)^{q-2} \psi\left(y, \phi(y), \int_{0}^{y} k(y, \sigma, \phi(\sigma)) d \sigma, \int_{0}^{T} g(y, \sigma, \phi(\sigma)) d \sigma\right) d y$

وبالتالي يكون حل المعادلتين (1.2) - (1.1) هو ايضا حل للمعادلة التكاملية : $\phi(t)=\delta+\gamma t+\frac{1}{\Gamma(q)} \int_{0}^{t}(t-y)^{q-1} \psi\left(y, \phi(y), \int_{0}^{y} k(y, \sigma, \phi(\sigma)) d \sigma, \int_{0}^{T} g(y, \sigma, \phi(\sigma)) d \sigma\right) d y$ $-\frac{t}{\Gamma(q-1)} \int_{0}^{T}(T-y)^{q-2} \psi\left(y, \phi(y), \int_{0}^{y} k(y, \sigma, \phi(\sigma)) d \sigma, \int_{0}^{T} g(y, \sigma, \phi(\sigma)) d \sigma\right) d y$

وهو المطلوب.

3- المبرهنات الاساسية للبحث

مبرهنة (3.1): (2) (ب)

في حالة تحقق الفرضيات (أ) - (ج) (ج) ،ولتكن (t) (t)

واذا $\quad n \quad L_{1}^{*}=\sup \left\{L_{1}(t): t \in[0, T]\right\}$

$n L_{1}^{*} T^{q}\left(\frac{1}{\Gamma(q)}+\frac{1}{\Gamma(q-1)}\right)<1$ فان المعادلتين $Q(\|x-y\|)<n\|x-z\|,(n>0)$

التكامليتين -التفاضليتين الكسريتين (1.2) - (1.1) تمتلك حلاً وحيدا على الفترة المغلقة [0,T (0, 
البرهان:

نعرف الدالة N :C $N \phi(t)=\delta+\gamma t+\frac{1}{\Gamma(q)} \int_{0}^{t}(t-y)^{q-1} \psi\left(y, \phi(y), \int_{0}^{y} k(y, \sigma, \phi(\sigma)) d \sigma, \int_{0}^{T} g(y, \sigma, \phi(\sigma)) d \sigma\right) d y$

$$
-\frac{t}{\Gamma(q-1)} \int_{0}^{T}(T-y)^{q-2} \psi\left(y, \phi(y), \int_{0}^{y} k(y, \sigma, \phi(\sigma)) d \sigma, \int_{0}^{T} g(y, \sigma, \phi(\sigma)) d \sigma\right) d y
$$

$$
E_{r}=\{\phi \in C,\|\phi\| \leq r\}
$$

بالإمكان اثبات ان الدالة N تمتلك نقطة ثابتة على المجموعة

وهذه النقطة الثابتة تمثل الحل للمعادلتين (1.2) - (1.1)، وعليه $\|N \phi(t)\| \leq|\delta|+|\gamma| t+\frac{1}{\Gamma(q)} \int_{0}^{t}(t-y)^{q-1}\left\|\psi\left(y, \phi(y), \int_{0}^{y} k(y, \sigma, \phi(\sigma)) d \sigma, \int_{0}^{T} g(y, \sigma, \phi(\sigma)) d \sigma\right)\right\| d y$ $\left.+\frac{t}{\Gamma(q-1)} \int_{0}^{T}(T-y)^{q-2} \| \psi\left(y, \phi(y), \int_{0}^{s} k(y, \sigma, \phi(\sigma)) d \sigma, \int_{0}^{T} g(y, \sigma, \phi(\sigma)) d \sigma\right)\right) \| d y$. $\leq|\delta|+|\gamma| t+\frac{1}{\Gamma(q)} \int_{0}^{t}(t-y)^{q-1} \| \psi\left(y, \phi(y), \int_{0}^{y} k(y, \sigma, \phi(\sigma)) d \sigma, \int_{0}^{T} g(y, \sigma, \phi(\sigma)) d \sigma\right)$ $-\psi(y, 0,0,0)+\psi(y, 0,0,0) \| d y$ $+\frac{t}{\Gamma(q-1)} \int_{a}^{T}(T-y)^{q-2} \| \psi\left(y, \phi(y), \int_{0}^{y} k(y, \sigma, \phi(\sigma)) d \sigma, \int_{0}^{T} g(y, \sigma, \phi(\sigma)) d \sigma\right)$ $-\psi(y, 0,0,0)+\psi(y, 0,0,0) \| d y$.

$\leq|\delta|+|\gamma| t+\frac{1}{\Gamma(q)} \int_{0}^{t}(t-y)^{q-1} \| \psi\left(y, \phi(y), \int_{0}^{y} k(y, \sigma, \phi(\sigma)) d \sigma, \int_{0}^{T} g(y, \sigma, \phi(\sigma)) d \sigma\right)$ $+\frac{t}{\Gamma(q-1)} \int_{0}^{T}(T-y)^{q-2} \| \psi\left(y, \phi(y), \int_{0}^{y} k(y, \sigma, \phi(\sigma)) d \sigma, \int_{0}^{T} g(y, \sigma, \phi(\sigma)) d \sigma\right)$ $-\psi(y, 0,0,0)\left\|d y+\frac{t}{\Gamma(q-1)} \int_{a}^{T}(T-y)^{q-2}\right\| \psi(y, 0,0,0) \| d y$. $\leq|\delta|+|\gamma| t+\frac{1}{\Gamma(q)} \int_{0}^{t}(t-y)^{q-1} m(y) Q\left(\|\phi(y)\|+\left\|\int_{0}^{y} k(y, \sigma, \phi(\sigma)) d \sigma\right\|\right.$ $\left.+\left\|\int_{0}^{T} g(y, \sigma, \phi(\sigma)) d \sigma\right\|\right) d y+\frac{t}{\Gamma(q-1)} \int_{0}^{T}(T-y)^{q-2} m(y) Q(\|\phi(y)\|$ $\left.+\left\|\int_{0}^{y} k(y, \sigma, \phi(\sigma)) d \sigma\right\|+\left\|\int_{0}^{T} g(y, \sigma, \phi(\sigma)) d t\right\|\right) d y$ $+\frac{1}{\Gamma(q)} \int_{0}^{t}(t-y)^{q-1}\|\psi(y, 0,0,0)\| d y+\frac{t}{\Gamma(q-1)} \int_{0}^{T}(T-y)^{q-2}\|\psi(y, 0,0,0)\| d y$. 


$$
\begin{aligned}
& \|N \phi(t)\| \leq|\delta|+|\gamma| t+\frac{1}{\Gamma(q)} \int_{0}^{t}(t-y)^{q-1} m(y) Q\left(\|\phi\|+h_{1}(y)\|\phi\|+u_{1}(y)\|\phi\|\right) d y \\
& +\frac{T}{\Gamma(q-1)} \int_{0}^{T}(T-y)^{q-2} m(y) Q\left(\|\phi\|+h_{1}(y) \phi+u_{1}(y)\|\phi\|\right) d y \\
& +\frac{N^{*}}{\Gamma(q)} \int_{0}^{t}(t-y)^{q-1} d y+\frac{T N^{*}}{\Gamma(q-1)} \int_{0}^{T}(T-y)^{q-2} d y . \\
& \|N \phi(t)\| \leq|\delta|+|\gamma| T+\frac{1}{\Gamma(q)} \int_{0}^{t}(t-y)^{q-1} m(y)\left(1+h_{1}(y)+u_{1}(y)\right) Q(\|\phi\|) d y \\
& +\frac{T}{\Gamma(q-1)} \int_{0}^{T}(T-y)^{q-2} m(y)\left(1+h_{1}(y)+u_{1}(y)\right) Q\|\phi\| d y \\
& +\frac{N^{*}}{\Gamma(q)} \int_{0}^{t}(t-y)^{q-1} d y+\frac{T N^{*}}{\Gamma(q-1)} \int_{0}^{T}(T-y)^{q-2} d y . \\
& \text { نضع } \\
& \|N \phi(t)\| \leq|\delta|+|\gamma| T+\frac{H}{\Gamma(q)} \int_{0}^{t}(t-y)^{q-1} Q(\|\phi\|) d y+\frac{T H}{\Gamma(q-1)} \int_{0}^{T}(T-y)^{q-2} Q(\|\phi\|) d y \\
& +\frac{N^{*}}{\Gamma(q)} \int_{0}^{t}(t-y)^{q-1} d y+\frac{N^{*} T}{\Gamma(q-1)} \int_{0}^{T}(T-y)^{q-2} d y \\
& \leq|\delta|+|\gamma| T+\frac{H Q(r)}{\Gamma(q)} \int_{0}^{t}(t-y)^{q-1} d y+\frac{N^{*}}{\Gamma(q)} \int_{0}^{t}(t-y)^{q-1} d y+\frac{N^{*} T}{\Gamma(q-1)} \int_{0}^{T}(T-y)^{q-2} d y \\
& +\frac{T H Q(r)}{\Gamma(q-1)} \int_{0}^{T}(T-y)^{q-2} d y \\
& \leq|\delta|+|\gamma| T+\frac{T^{q} H Q(r)}{\Gamma(q+1)}+\frac{N^{*} T^{q}}{\Gamma(q+1)}+\frac{H Q(r) T^{q}}{\Gamma(q)}+\frac{N^{*} T^{q}}{\Gamma(q)} . \\
& \leq|\delta|+|\gamma| T+T^{q} H Q(r)\left(\frac{1}{\Gamma(q)}+\frac{1}{\Gamma(q+1)}\right)+N^{*} T^{q}\left(\frac{1}{\Gamma(q)}+\frac{1}{\Gamma(q+1)}\right) \\
& =|\delta|+|\gamma| T+\left(H Q(r)+N^{*}\right) T^{q}\left(\frac{1}{\Gamma(q)}+\frac{1}{\Gamma(q+1)}\right)=l \text {. }
\end{aligned}
$$

لذا بعد ذلك، نستعمل مبدأ انكماش باناخ لإثبات أن $N$ هو $N$ هو انكماش. ليكن 


$$
\begin{aligned}
& \|N x(t)-N z(t)\| \leq \frac{1}{\Gamma(q)} \int_{0}^{t}(t-y)^{q-1} \| \psi\left(y, x(y), \int_{0}^{y} k(y, \sigma, x(\sigma)) d \sigma, \int_{0}^{T} g(y, \sigma, x(\sigma)) d \sigma\right) \\
& \left.-\psi\left(y, z(y), \int_{0}^{y} k(y, \sigma, z(\sigma)) d \sigma, \int_{0}^{T} g(y, \sigma, z(\sigma)) d \sigma\right)\right) \| d y \\
& +\frac{t}{\Gamma(q-1)} \int_{0}^{T}(T-y)^{q-2} \| \psi\left(y, x(y), \int_{0}^{y} k(y, \sigma, x(\sigma)) d \sigma, \int_{0}^{T} g(y, \sigma, x(\sigma)) d \sigma\right) \\
& \left.{ }_{-} \psi\left(y, z(y), \int_{0}^{y} k(y, \sigma, z(\sigma)) d \sigma, \int_{0}^{T} g(y, \sigma, z(\sigma)) d \sigma\right)\right) \| d y . \\
& \text { وباستعمال الفرضيات( أ)- (ج)، نحصل على: } \\
& \|N x(t)-N z(t)\| \leq \frac{1}{\Gamma(q)} \int_{0}^{t}(t-y)^{q-1} m(y) Q\left(\|x(y)-z(y)\|+\left\|\int_{0}^{y} k(y, \sigma, x(\sigma)) d \sigma-\int_{0}^{y} k(y, \sigma, y(\sigma)) d \sigma\right\|\right. \\
& \left.+\left\|\int_{0}^{T} g(y, \sigma, x(\sigma)) d \sigma-\int_{0}^{T} g(y, \sigma, z(\sigma)) d \sigma\right\|\right) d y+\frac{t}{\Gamma(q-1)} \int_{0}^{T}(T-y)^{q-2} m(y) Q(\|x(y)-z(y)\| \\
& \left.+\left\|\int_{0}^{y} k(y, \sigma, x(\sigma)) d \sigma-\int_{0}^{y} k(y, \sigma, z(\sigma)) d \sigma\right\|+\left\|\int_{0}^{T} g(y, \sigma, x(\sigma)) d \sigma-\int_{0}^{T} g(y, \sigma, z(\sigma)) d \sigma\right\|\right) d y . \\
& \leq \frac{1}{\Gamma(q)} \int_{0}^{t}(t-y)^{q-1} m(y) Q(\|x-z\|+h(y)\|x-z\|+u(y)\|x-z\|) d y \\
& +\frac{t}{\Gamma(q-1)} \int_{0}^{T}(T-y)^{q-2} m(y) Q(\|x-z\|+h(y)\|x-z\|+u(y)\|x-z\|) d y . \\
& \|N x(t)-N z(t)\| \leq \frac{1}{\Gamma(q)} \int_{0}^{t}(t-y)^{q-1} m(y)(1+h(y)+u(y)) Q(\|x-z\|) d y \\
& +\frac{t}{\Gamma(q-1)} \int_{0}^{T}(T-y)^{q-2} m(y)(1+h(y)+u(y)) Q(\|x-z\|) d y . \\
& \|N x(t)-N z(t)\| \leq \frac{L_{1}^{*} n}{\Gamma(q)}\|x-z\| \int_{0}^{t}(t-y)^{q-1} d y+\frac{T L_{1}^{*} n}{\Gamma(q-1)}\|x-z\| \int_{0}^{T}(T-y)^{q-2} d y \\
& =\frac{T^{q} L_{1}^{*} n}{\Gamma(q+1)}\|x-y\|+\frac{T^{q} L_{1}^{*} n}{\Gamma(q)}\|x-y\| \text {. } \\
& =T^{q} L_{1}^{*} q\left(\frac{1}{\Gamma(q)}+\frac{1}{\Gamma(q+1)}\right)\|x-y\| .
\end{aligned}
$$

مما سبق، وباتباع مبدأ الانكماش الاساسي، نستتتج ان N Nو تطبيق انكماشي، وحسب مبرهنة باناخ للنقطة الثابتة فيوجد نقطة وحيدة للتطبيق N مثئل حل لمسالة القيم الحدودية (1.2)-(1.1).

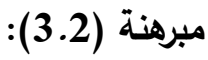

لتكن الفرضيات (أ)-(ج) متحققة وليكن |(3) 
$\left\|\psi\left(y, \phi(y), \int_{0}^{y} k(y, \sigma, \phi(\sigma)) d \sigma, \int_{0}^{T} g(y, \sigma, \phi(\sigma)) d \sigma\right)\right\| \leq \omega(t)$.

$\omega(t) \in L(J)$

فان مسألة القيم الحدودية (1.2. (1.2)- (1.1) تمتلك على الاقل حلاً واحداً على الفترة [0,T]. البرهان: تامل $(R x)(t)=\frac{1}{\Gamma(q)} \int_{0}^{t}(t-y)^{q-1} \psi\left(y, x(y), \int_{0}^{y} k(y, \sigma, x(\sigma)) d \sigma, \int_{0}^{T} g(y, \sigma, x(\sigma)) d \sigma\right) d y$. $\left.(S x)(t)=\frac{t}{\Gamma(q-1)} \int_{0}^{T}(T-y)^{q-2} \psi\left(y, x(y), \int_{0}^{y} k(y, \sigma, x(\sigma)) d \sigma, \int_{0}^{T} g(y, \sigma, x(\sigma)) d \sigma\right)\right) d y+\not t+\delta$. من الملاحظ انه اذا كانت $R x_{1}, S x_{2} \in E_{r} \quad$ فان $x_{1}, x_{2} \in E_{r}$ $\left\|R x_{1}+S x_{2}\right\|=\| \frac{1}{\Gamma(q)} \int_{0}^{t}(t-y)^{q-1} \psi\left(y, x_{1}(y), \int_{0}^{y} k\left(y \sigma, x_{1}(\sigma)\right) d \sigma, \int_{0}^{T} g\left(y, \sigma, x_{1}(\sigma)\right) d \sigma\right) d y$ $\left.+\frac{t}{\Gamma(q-1)} \int_{0}^{T}(T-y)^{q-2} \psi\left(y, x_{2}(y), \int_{0}^{y} k\left(y, \sigma, x_{2}(\sigma)\right) d \sigma, \int_{0}^{T} g\left(y, \sigma, x_{2}(\sigma)\right) d \sigma\right)\right) d y \|+|\gamma| t$. $\left.\leq \frac{1}{\Gamma(q)} \int_{0}^{t}(t-y)^{q-1} \| \psi\left(y, x_{1}(y), \int_{0}^{y} k\left(y, \sigma, x_{1}(\sigma)\right) d \sigma, \int_{0}^{T} g\left(y, \sigma, x_{1}(\sigma)\right) d \sigma\right)\right) \| d y$ $\left.+\frac{t}{\Gamma(q-1)} \int_{0}^{T}(T-y)^{q-2} \| \psi\left(y, x_{2}(y), \int_{0}^{y} k\left(y, \sigma, x_{2}(\sigma)\right) d \sigma, \int_{0}^{T} g\left(y, \sigma, x_{2}(\sigma)\right) d \sigma\right)\right) \| d y+|\gamma| t$.

$$
\begin{aligned}
& \leq \frac{\|\phi\|_{L}}{\Gamma(q)} \int_{0}^{t}(t-y)^{q-1} d y+\frac{T\|\phi\|_{L}}{\Gamma(q-1)} \int_{0}^{T}(t-y)^{q-2} d y+|\gamma| T . \\
& \leq \frac{\|\phi\|_{L} T^{q}}{\Gamma(q+1)}+\frac{T^{q}\|\phi\|_{L}}{\Gamma(q)} . \\
& \leq\left(\frac{1}{\Gamma(q)}+\frac{1}{\Gamma(q+1)}\right) T^{q}\|\phi\|_{L} .
\end{aligned}
$$

الان، سنبر هن على ان Sx هو انكماش

$$
\left\|S x_{1}-S x_{2}\right\| \leq \frac{t}{\Gamma(q-1)} \int_{0}^{T}(T-y)^{q-2} \| \psi\left(y, x_{1}(y), \int_{0}^{y} k\left(y, \tau, x_{1}(\tau)\right) d \tau, \int_{0}^{T} g\left(y, \tau, x_{1}(\tau)\right) d \tau\right)
$$$$
\left.-\psi\left(y, x_{2}(y), \int_{0}^{y} k\left(y, \tau, x_{2}(\tau)\right) d \tau, \int_{0}^{T} g\left(y, \tau, x_{2}(\tau)\right) d \tau\right)\right) \| d y .
$$

$\leq \frac{t}{\Gamma(q-1)} \int_{0}^{T}(T-y)^{q-2} m(y)(1+h(y)+u(y)) \phi\left(\left\|x_{1}-x_{2}\right\|\right) d y$ 


$$
\begin{aligned}
& \left\|S x_{1}-S x_{2}\right\| \leq \frac{t}{\Gamma(q-1)} \int_{0}^{T}(T-y)^{q-2} L_{1}^{*} n\left\|x_{1}-x_{2}\right\| d y . \\
& \leq \frac{n L_{1}^{*}}{\Gamma(q)} T^{q}\left\|x_{1}-x_{2}\right\| \text {. } \\
& \text { من الواضح ان S Sو نطبيق انكماش، وبما انه (t) مستمرة، فان Rx دالة مستمرة. } \\
& \|R x(t)\|=\left\|\frac{t}{\Gamma(q)} \int_{0}^{t}(t-y)^{q-1} f\left(y, x(y), \int_{0}^{y} k(y, \sigma, x(\sigma)) d \sigma, \int_{0}^{T} g(y, \sigma, x(\sigma)) d \sigma\right) d y\right\| \text {. } \\
& \leq\|\phi\|_{L} \int_{0}^{t} \frac{(t-y)^{q-1}}{\Gamma(q)} d y . \\
& \|R x(t)\|=\frac{T^{q}\|\phi\|_{L}}{\Gamma(q+1)}
\end{aligned}
$$

وبالتالي، فان R هي مقيدة بانتظام على E $E_{r}$. الان سنبرهن أن (Rx(t) متساوي الاستمرارية. . $t_{1}<t_{2}, x \in E_{r} g t_{1}, t_{2} \in[0, T]$ تلكن وباعتبار ان $\psi$ $\sup _{(t, y) \in J \times E_{r}}\left\|\psi\left(y, x(y), \int_{0}^{y} k(y, \sigma, x(\sigma)) d \sigma, \int_{0}^{T} g(y, \sigma, x(\sigma)) d \sigma\right)\right\|=c<\infty$.

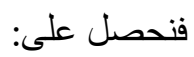

$$
\begin{aligned}
& \left\|R x\left(t_{1}\right)-R x\left(t_{2}\right)\right\|=\| \frac{1}{\Gamma(q)} \int_{0}^{t_{1}}\left(t_{1}-y\right)^{q-1} \psi\left(y, x(y), \int_{0}^{y} k(y, \sigma, x(\sigma)) d \sigma, \int_{0}^{T} g(y, \sigma, x(\sigma)) d \sigma\right) d y \\
& -\frac{1}{\Gamma(q)} \int_{0}^{t_{2}}\left(t_{2}-y\right)^{q-2} \psi\left(y, x(y), \int_{0}^{y} k(y, \sigma, x(\sigma)) d \sigma, \int_{0}^{T} g(y, \sigma, x(\sigma)) d \sigma\right) d y \| . \\
& \leq \frac{1}{\Gamma(q)} \| \int_{0}^{t_{1}}\left[\left(t_{1}-y\right)^{q-1}-\left(t_{2}-y\right)^{q-2}\right] \psi\left(y, x(y), \int_{0}^{y} k(y, \sigma, x(\sigma)) d \sigma, \int_{0}^{T} g(y, \sigma, x(\sigma)) d \sigma\right) d y \\
& +\left\|\int_{t_{1}}^{t_{2}}\left(t_{2}-s\right)^{q-2} \psi\left(y, x(y), \int_{0}^{y} k(y, \sigma, x(\sigma)) d \sigma, \int_{0}^{T} g(y, \sigma, x(\sigma)) d \sigma\right) d y\right\| . \\
& \leq \frac{c}{\Gamma(q+1)}\left[2\left(t_{2}-t_{1}\right)^{q}+\left(t_{1}^{q}-t_{2}^{q}\right)\right] \\
& \text { لذلك فان R متراص محليا، وباستعمال مبرهنة Arzela - Ascoli، فان R يعتبر متراصاً. }
\end{aligned}
$$

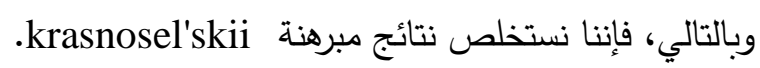$$
\text { - }
$$

1) ZiBai H.LU., "positive Solutions for boundary Value Problems of nonlinear fractional differential equation", J, Mat. Anal . Appl. 311, 445-505, (2005).

2) Kilbas A.A., Srivastava H.M. and Trujillo Juan J., "Theory and applications of fractional differential equations", North-Holland Mathematils studies, 204. Elsevies Science B.V., Amsterdam, 2006.

3) Lakshmi kantham V., Leela S. and Vasundhara J., "Theary of Fractional Dynamic Systems", Cambridye. Academic Pablishers, Cambridge, 2009.

4) Momani S.M., "Local and global existence theorems on fractional integrodifferential equations", Journal of Fractional Calculus, Vol, 18, pp.81-86, 2000. 
5) Podlubny I., "Fractional Differential Equations", Academic Press, San Diego, 1999.

6) Momani S.M. and Hadid S.B., "on the inequalities of integro-differential fractional equations", Interenational Journal of Applied Mathematics, Vol.12, no.1, pp.29-37, 2003.

7) Momani S.M and El- khazali R., "On the existence of existence of extremal solutions of the fractional integro-elifferential equations", J.Fractional Calculus, Vol.18, 87-92, (2000). 